\title{
La investigación-acción participativa como método para un nuevo modelo de gobernanza en el ámbito de la inclusión en Gipuzkoa
}

\author{
Cinta Guinot Viciano \\ Departamento Trabajo Social y Sociología, Universidad de Deusto \\ <cguinot@deusto.es>
}

\author{
Ane Ferran Zubillaga \\ Departamento Trabajo Social y Sociología, Universidad de Deusto \\ Asun Berasategui Otegui \\ Departamento Trabajo Social y Sociología, Universidad de Deusto
}

Artikulu honetan, gizarte-bazterkeriaren esparruan Gipuzkoan inplikaturik dauden eragileen partehartzerako eredu baten proposamena aurkezten da, Deustuko Unibertsitateak Gipuzkoako Foru Aldundiarekin duen hitzarmenaren testuinguruan egina, gobernantza prozesu bat diseinatzen eta dinamizatzen lagundu dezan, eragileen arteko elkarrekintza sustatuz eta ekarpenak sistematizatzen lagunduz, era horretan, elkarrekin definitutako arazoei aurre egiteko erantzunak bilatzeko asmoz, elkar-eraikuntza prozesu bat bultzatuz. Proposaturiko metodologia Ikerketa ekintza parte-hartzailean oinarritua dago, eta ondorengo hiru funtsezko printzipioetatik abiatuz garatu da: ezagutzaren elkar-sorkuntza; gailentzen doan prozesua; eta partaide guztien ekarpenetarako errespetua. Parte-hartze esperientzia bat da, eta lantzen da gizarte-bazterkeriaren esparruan Gipuzkoan inplikaturik dauden eragileen arteko gobernantza eredu berrien diseinua, garapena eta jarraipena.

\section{GAKO-HITZAK:}

Gobernantza, gizarte-bazterketa, ikerketa ekintza parte-hartzailea, Gipuzkoa..
En este artículo se presenta una propuesta de modelo de participación de los agentes implicados en el ámbito de la inclusión social en Gipuzkoa, elaborada en el marco del convenio de colaboración que la Universidad de Deusto tiene con la Diputación Foral de Gipuzkoa para contribuir a diseñar y dinamizar un proceso de gobernanza, facilitando la interacción entre los agentes, y ayudándoles a sistematizar sus aportaciones y favoreciendo un proceso de co-construcción orientado a buscar respuestas a los problemas que se definan conjuntamente. La metodología propuesta está basada en la investigación-acción participativa (IAP), que se desarrolla a partir de tres principios básicos: co-generación del conocimiento; proceso emergente; y respeto a la participación y aportaciones de todas y todos los participantes. Se trata de una experiencia participativa en el diseño, desarrollo y seguimiento de nuevos modelos de gobernanza entre los agentes implicados en el ámbito de la inclusión en Gipuzkoa.

\section{Palabras Clave:}

Gobernanza, inclusión social, investigación-acción participativa, Gipuzkoa. 


\section{Contextualización de la experiencia ${ }^{1}$}

De todos es sabido que el sistema vasco de servicios sociales es un sistema de atención fragmentado entre los tres niveles administrativos presentes en la Comunidad Autónoma del País Vasco (CAPV): Gobierno Vasco, diputaciones forales y municipios. Un sistema cuya articulación en los diversos niveles de atención fue establecida primero en el Decreto 155/2001, de Determinación de Funciones en Materia de Servicios Sociales, y recogida después en el texto de la propia Ley $12 / 2008$, de Servicios Sociales. En esta estructura, se distinguen las prestaciones del sistema de garantía de ingresos (en manos del Gobierno Vasco), de la atención primaria (en manos de los municipios) y de la atención secundaria (de competencia foral). Uno de los criterios utilizados para determinar el reparto competencial entre la atención municipal y foral se da a partir de la consideración de la necesidad de una mayor o menor intensidad del apoyo prestado a la persona usuaria.

Lo que se ha denominado fragmentación del sistema vasco de servicios sociales genera alguna distorsión, no sólo en el ámbito de la comunidad autónoma sino incluso dentro del propio territorio histórico de Gipuzkoa, entre el ámbito municipal y foral. Ambos niveles son conscientes de su interdependencia y constatan la necesidad de establecer un diálogo para acordar o consensuar los límites o las fronteras en la intervención de cada nivel, así como los espacios convergentes, o para identificar los elementos clave en los procesos de ayuda en los itinerarios establecidos con cada persona atendida. Conviene tener en cuenta al respecto que las personas van y vienen, llegan y se quedan, sin preguntar si su referencia está en la atención primaria o en la atención secundaria. Esta necesidad de abordar los procesos de ayuda de manera global e integral, poniendo en el centro a cada una de las personas atendidas, requiere de un diálogo entre ambos niveles administrativos, diálogo que debería darse tanto en el ámbito político como técnico.

Esta dificultad en la articulación competencial también es identificada por el resto de los agentes del sistema. Así, el estudio elaborado por el SIIS Centro de Documentación y Estudios (2015) sobre la reordenación de la red de recursos y programas para la inclusión social en Gipuzkoa recoge la opinión de diversos agentes guipuzcoanos en torno al hecho de que, en la medida que el sistema se va complejizando, la capacidad de algunos ayuntamientos de responder a las demandas y exigencias planteadas por el marco legal es muy limitada, debido a su tamaño y capacidad, así como a la cantidad de recursos necesarios.

Esta realidad no es nueva ni desconocida; ya fue presentada profusamente en un informe

${ }^{1}$ Este artículo está basado en una comunicación presentada por las autoras en el VI Congreso de la Red Española de Política Social (Sevilla, 2016). extraordinario del Ararteko (2010) que consideraba imprescindible subrayar el desajuste existente entre las funciones atribuidas a los servicios municipales y los recursos técnicos y humanos puestos a disposición de los municipios para poder cumplirlas. Siete años más tarde, el informe extraordinario realizado por el Ararteko (2016) respecto a la situación actual de los servicios sociales municipales en la CAPV plantea una serie de conclusiones y recomendaciones que también destacan algunas de las dificultades y retos que los servicios sociales municipales todavía afrontan para implementar las indicaciones de la Ley 12/2008.

Respecto a la valoración general de la situación y las principales dificultades manifestadas por las personas que han participado en este estudio, cabe señalar, en primer lugar el hecho de que se detecta de manera unánime una falta de desarrollo normativo que, en consecuencia, ralentiza la toma de decisiones, al tiempo que limita la puesta en marcha de estrategias adecuadas para buscar soluciones a los problemas que se dan en el nivel de atención primaria.

En segundo lugar, se plantea otra dificultad en relación con la falta de un modelo común y consensuado en el ámbito de la atención primaria. Además, en el mismo informe se diagnostica la existencia de obstáculos que dificultan que los ayuntamientos puedan cumplir con las funciones y competencias indicadas en la Ley de Servicios Sociales, de manera que es difícil asumir el conjunto de los servicios de atención primaria entendidos, tal y como establece la Ley, como un continuo integrado de atención que va más allá de la información, el diagnóstico y la orientación.

Por último, desde esta perspectiva general, también se observa una pérdida progresiva del gasto municipal en servicios sociales en relación con el conjunto de gasto público en este ámbito, por lo que aunque se produce un incremento en las necesidades que atienden los servicios municipales, no se ha aumentado la asignación de los recursos económicos municipales.

En cuanto a la valoración del desarrollo normativo y la situación de los diferentes servicios de atención primaria, el informe extraordinario (Ararteko, 2016) plantea, entre otras, las siguiente conclusiones:

- La importancia de la aprobación del Decreto de Cartera (Decreto 185/2015) y del Mapa de Servicios Sociales para clarificar competencias y responsabilidades de cada nivel institucional, que posibilitará reducir la actual heterogeneidad en la dotación de recursos en los diferentes municipios.

- No obstante, se manifiesta el riesgo de que determinadas necesidades sociales no sean cubiertas adecuadamente por los servicios y prestaciones establecidas por el Decreto de Cartera, existiendo servicios, como los de carácter preventivo o los destinados a las 
personas en riesgo de dependencia, exclusión o desprotección, que han sido menos desarrollados y requieren de un mayor impulso.

- De otro lado, se hace manifiesta la necesidad de avanzar en el desarrollo e implementación de herramientas comunes de diagnóstico y valoración para optimizar los procesos de intervención con las personas atendidas en el ámbito de los servicios sociales de atención primaria, siendo necesario repensar el uso de estas herramientas, especialmente en lo referente al ámbito de la exclusión.

Un último elemento que destacar en las conclusiones de este informe extraordinario es la constatación de dificultades de coordinación entre los servicios sociales de atención primaria y secundaria, haciéndose, por tanto, necesaria la tarea de delimitar funciones y construir sinergias entre los dos niveles de intervención, todo ello a fin de mejorar la atención de las personas atendidas, de modo que sea posible superar divergencias y concretar las funciones de la atención especializada y primaria a escala territorial.

Por otro lado, los efectos de la crisis económicosocial que padecemos desde 2008 han puesto de manifiesto cuestiones clave para avanzar hacia la consecución de mayores cotas de bienestar y cohesión social:

- En primer lugar, la necesidad de que los poderes públicos cumplan y hagan cumplir los derechos socioeconómicos promulgados.

- En segundo, la necesidad de profundizar en modelos de ciudadanía que incidan en la idea del deber ciudadano o de la solidaridad cívica como criterios básicos articuladores de cohesión social.

- Por último, la importancia otorgada a la necesidad de buscar alianzas que generen sinergias y que permitan avanzar en la resolución de las situaciones de desigualdad y en la consecución de la justicia social.

En cualquier caso, si para afrontar los retos de futuro que se abren tras la crisis hay que buscar alianzas -dado que, como dice García Roca (2016), la lucha contra la exclusión requiere de la participación de todos los agentes, porque ninguno de ellos, por sí sólo, puede afrontarla con éxito-, se hace imprescindible determinar los modos de colaboración entre los distintos actores, los cuales estarán sujetos al contexto sociopolítico de cada momento.

Para ello, es fundamental articular propuestas que enraícen la política social desde enfoques que partan, en primer lugar, de la consideración de las capacidades personales y comunitarias, no sólo como recursos, sino también como ejercicio real de oportunidades, tal y como plantea Sen (2010). En segundo lugar, deben entroncarse en el enfoque comunitario, un enfoque que, en palabras de Fantova
(2014), impulse una visión de suma positiva, donde los agentes involucrados en el cuidado y la atención a todas las personas establezcan relaciones sinérgicas, promoviendo, entre todas y todos, capacidades, recursos, vínculos y procesos que, de hecho, ya existen en la propia comunidad. Y en tercer lugar, que se basen en modelos de gobernanza participativa real y efectiva que derivan de la consideración del Estado relacional (Alguacil, cit. en Jaraíz, 2009), aquel orientado desde la cultura alter-activa y de co-liderazgo, que pretende no tanto la colaboración con, sino la colaboración para, por lo que el espacio de relación será diverso, definible en cada caso y abierto al aporte de entidades con capacidades diversas. Se trata, en definitiva, de estructurar nuevas formas de gobernanza.

\section{Nuevos modelos de gobernanza para articular políticas de inclusión con futuro}

Cuando hablamos de gobernanza, nos encontramos con un concepto polisémico y todavía ambiguo, que hace referencia a los nuevos modos de ejercer el gobierno por parte de las instituciones encargadas en contextos de creciente complejidad. Así, siguiendo a Blanco y Gomà (2006) el concepto de gobernanza nos vincula a la idea de la interacción entre los actores que actúan en un contexto dado (en nuestro caso, en el ámbito de la inclusión social en el territorio de Gipuzkoa) y en el que se saben interdependientes.

La Diputación Foral de Gipuzkoa (DFG) ha iniciado la implantación de un modelo de gobernanza basado en la colaboración como eje de su estrategia para hacer frente a los retos que afronta el territorio en el presente y a futuro. Parte de la necesidad de un liderazgo compartido con la sociedad, en el que las instituciones tienen que ser parte, no pueden actuar solas y en exclusiva, entendiendo que los problemas que afectan a la sociedad necesitan de soluciones compartidas. Desde esta perspectiva, se ha puesto en marcha el programa Etorkizuna Eraikiz (Construyendo el Futuro), herramienta para afrontar con garantías los retos del territorio. Dicha experiencia se apoya en dos pilares:

- GipuzkoaLab, dentro del cual se están poniendo en práctica experiencias piloto sobre proyectos de futuro.

- Gipuzkoa Taldean, con el que se ha puesto en marcha una reflexión compartida con las personas y los agentes de la sociedad organizada sobre qué proyectos van a impulsarse durante los próximos diez años, dentro de un modelo de colaboración público-privada.

En este contexto, las cuatro universidades con sede en Gipuzkoa participan en la plataforma Ikergune para la generación de conocimiento en aquellas áreas sobre las que se experimente en GipuzkoaLab o que se estimen necesarias desde la perspectiva estratégica de Gipuzkoa Taldean, con actividades de 
investigación, debate y divulgación. La experiencia que vamos a narrar se ubica en la participación de la Universidad de Deusto (UD) en esta plataforma en las áreas que afectan a la política social guipuzcoana.

La referencia a la necesidad de establecer nuevos modelos de gobernanza para la atención a las necesidades sociales de las y los ciudadanos guipuzcoanos ha sido una constante desde la publicación de la tercera Ley vasca de Servicios Sociales. La diputada de Política Social de la DFG en la legislatura 2007-2011 Maite Etxaniz, tras analizar la relación pública-privada en la prestación de los servicios sociales en el territorio histórico de Gipuzkoa, consideraba que podía identificarse un 'modelo guipuzcoano' de prestación de servicios sociales:

Se ha hablado de la complejidad de la sociedad guipuzcoana. Una sociedad que ha sabido ser solidaria, una sociedad en la que las personas son el centro de la acción diaria, tanto de los colectivos como de las instituciones. Es esta realidad la que ha configurado lo que se ha venido llamando 'el modelo guipuzcoano', establecido sobre la base de una estrecha colaboración entre la administración pública y el Tercer Sector. Por ello, Gipuzkoa demanda también una nueva cultura política y de la gobernanza, que se apoye en la colaboración público-privada y la implicación y participación de la ciudadanía en las políticas públicas (Etxaniz, 2009: 29-30).

Un modelo que el Gobierno foral actual también recoge en su Plan Estratégico de Gestión para el periodo 2015-2019, que propone un:

Modelo de Gobernanza abierto y colaborativo, basado en las personas y en el liderazgo transformacional y compartido; la colaboración público-privada para el impulso de los proyectos estratégicos; la interacción y deliberación permanente con la ciudadanía y el resto de agentes económicos y sociales del Territorio, y la participación activa y protagonista de los equipos político y técnico de Diputación Foral de Gipuzkoa (Diputación Foral de Gipuzkoa, 2015: 6).

Uno de los proyectos estratégicos que recoge este plan es, precisamente, el Mapa de Servicios Sociales de Gipuzkoa, cuyo objetivo es:

Fortalecer nuestro modelo de bienestar social a través de la mejora del servicio, la mejora del equilibrio territorial, la creación de empleo y la complementariedad de la red natural de apoyo en colaboración con los ayuntamientos y las entidades sociales (Diputación Foral de Gipuzkoa, 2015: 18).

Este proyecto estratégico se articula, entre otros, en el objetivo que pretende analizar e implantar nuevas fórmulas de colaboración público-privada para ofrecer unos servicios sociales de calidad sostenibles económicamente (Diputación Foral de Gipuzkoa,
2015: 31). Se trata de un plan que requiere, según sus relatores:

De la máxima implicación y coordinación interinstitucional, en cuanto que plantea líneas de trabajo y proyectos que requieren la concertación con el resto de las instituciones, especialmente Gobierno Vasco, resto de Diputaciones Forales y Ayuntamientos (ibídem: 37).

Es más, el propio Plan Estratégico señala expresamente cómo ha de construirse el nuevo modelo de gobernanza que la institución foral quiere implantar y que constituye la base de la experiencia que vamos a relatar:

En relación al ámbito municipal y comarcal, se establecerán mecanismos específicos de trabajo conjunto y coordinación. En concreto, en algunos proyectos se tomará como referencia la experiencia de Gipuzkoa Sarean, que ha permitido definir a lo largo de los últimos seis años un modelo de gobernanza que, con la participación de múltiples y variados agentes públicos y privados, ha permitido compartir y desarrollar proyectos en común basados en la confianza entre dichos agentes. Con la base de dicho modelo de gobernanza, se procurará abordar líneas de trabajo del Plan que impliquen la participación de los municipios y, en su caso, de las agencias comarcales de desarrollo (Diputación Foral de Gipuzkoa, 2015: 37).

En lo que respecta a esta cuestión, suele ser el ámbito municipal quien reclama más claramente la existencia de un nuevo modelo de gobernanza (SIIS Centro de Documentación y Estudios, 2015) en el que se estructuren espacios en común, 'nodos de red' (Blanco y Gomà, 2006), que sean liderados por la red pública. En cualquier caso, es evidente la unanimidad que esta cuestión concita en todo el sistema guipuzcoano, si atendemos a que el propio Plan de Inclusión 2016-2020 presentado por el Departamento de Políticas Sociales de la DFG, en junio de 2016, señala la misión del Departamento como:

Cumplir con la responsabilidad pública de la Diputación Foral de Gipuzkoa de garantizar los derechos de la ciudadanía, dentro del marco competencial del sistema de servicios sociales, para contribuir a la mejora de las condiciones de vida de las personas y a la cohesión social en Gipuzkoa, desarrollando esta labor en colaboración con otros agentes sociales (Diputación Foral de Gipuzkoa, 2016: 31).

Una misión que se concreta en el primer punto de la visión estratégica del propio Departamento:

Ser una institución que, junto a los ayuntamientos, organizaciones del Tercer Sector, otras administraciones públicas y otros colaboradores, promueve la construcción de una red integrada y compartida de servicios sociales 
en el territorio, y que, con su actuación, refuerza el tejido social, la solidaridad y la cohesión social en Gipuzkoa (ibídem: 31).

En el marco de la misión y visión del Departamento de Políticas Sociales, el Plan de Inclusión asume como objeto fundamental "liderar construyendo entre diferentes e impulsando estrategias eficientes hacia la inclusión social" (ibídem, 2016: 34). La propia denominación del plan Elkar-EKIN (juego de palabras en euskara que significa 'trabajar juntos', 'en conjunto') quiere destacar esta idea de acción en común

\section{Construyendo ágoras. Espacios de co-construcción y co-generación de respuestas ante la inclusión}

Como señalábamos anteriormente, el Plan de Inclusión de Gipuzkoa 2016-2020 identifica un determinado modelo de gobernanza basado en la experiencia de Gipuzkoa Sarean. Se trata de un proyecto de investigación-acción liderado por la DFG, cuyo objetivo es la construcción de una nueva estructura de relaciones que faciliten el desarrollo de un nuevo modelo territorial en Gipuzkoa. Concretamente y en relación con el asunto que nos ocupa, busca crear espacios para la colaboración entre responsables políticos y técnicos de las comarcas, la DFG y de otros niveles administrativos.

El proyecto Gipuzkoa Sarean, desarrollado por la DFG y Orkestra (Centro de Investigación en Competitividad Territorial y Políticas, impulsado por la Universidad de Deusto) durante los últimos siete años, tiene su base conceptual en el modelo de la investigaciónacción participativa (IAP) recogido en Karlsen y Larrea (2015), que entienden como "una estrategia para el cambio en tiempo real en el que los elementos investigación, acción y participación se combinan y conectan en el mismo proceso" (ibídem: 26).

La IAP se define como un "método de investigación y aprendizaje colectivo de la realidad, basado en un análisis crítico con la participación activa de los grupos implicados, que se orienta a estimular la práctica transformadora y el cambio social" (Eizagirre y Zabala, 200o). Se trata de un enfoque de intervención donde se conjuga en un todo la investigación y la acción, superando dicotomías clásicas como la de sujeto-objeto, acción-reflexión, teoría-práctica. En él se combinan dos procesos, el de conocer y el de actuar, cada uno de ellos con lógicas y dinámicas distintas pero complementarias, que se nutren y retroalimentan, generando un proceso de cambio y una práctica transformadora. Seguimos a Iñiguez (1999, cit. en Ariño y Ferran, 2008: 73) cuando defendemos que los supuestos básicos sobre los que se sustenta la IAP son:

Que las personas construyen la realidad en la que viven; que las comunidades y los grupos en las que se inserta la acción investigadora tienen su propio desarrollo histórico y cultural, es decir, preceden a y prosiguen después de la investigación; que la relación entre investigador/a y personas de la comunidad o grupo ha de ser horizontal y dialógica, donde el diálogo es a la vez una categoría social y epistemológica; que toda comunidad dispone de los recursos necesarios para su evolución y transformación; que la investigación es propiedad de las personas investigadas; que la persona que investiga ejerce un rol de persona que se inserta en una comunidad; y finalmente que el proceso de investigación debe romper la relación de dependencia intelectual y sustituirla por un modelo de relación horizontal con las personas investigadas.

Los enfoques desde los que se aplica la IAP son diversos, afectados por la realidad en la que se desarrolla. En cualquier caso, la aplicación de este método se asienta sobre cinco principios básicos (Colectivo loé, 2003):

1. Las protagonistas de todo el proceso son las personas, estableciéndose entre ellas una relación de sujeto a sujeto.

2. El cambio se plantea a partir de las demandas o necesidades sentidas, dado que es éste el elemento que garantiza situar a las personas como protagonistas indiscutibles, sujetos de la transformación.

3. Es indispensable unir la teoría y la práctica como estrategia que ayuda a superar el verbalismo y el activismo.

4. Todo ello supone comprender la realidad como un todo integrado, concreto y complejo a la vez, por lo que tanto el análisis como la acción deben situarse complementariamente; es decir, se trata de intentar la articulación de los niveles micro y macro.

5. Por último, la IAP aboga por la transformación, una actitud comprometida de las y los sujetos participantes, que hacen suyo el axioma de Freire de que si el conocimiento no implica transformar la realidad, no es verdadero conocimiento.

Estamos, pues, ante un tipo de investigación social desarrollada por un equipo formado por investigadoras e investigadores y miembros de una organización, comunidad o red que tiene por objetivo mejorar la situación de las y los participantes y contribuir al debate académico. Se trata de una estrategia hacia la práctica, hacia el cambio. No se trata de una receta para procesos participativos, ni una solución fácil o rápida para gestionar la participación. El reto es implementar una nueva gobernanza que permita construir un 'nosotros', gestionando el conflicto que permite el tránsito desde el diagnóstico a la acción.

Para ello, sería necesario construir espacios de comunicación, lo que Karlsen y Larrea denominan 'ágora' (Karlsen y Larrea, 2015: 31). Se trata de 
un espacio común en el que se reúnen diferentes actores para enmarcar y definir problemas sociales, siempre y cuando no se pierda de vista la perspectiva de totalidad (público-privado, político-técnico, científico-práctico), y en el que se negocie lo que va a ser considerado como una solución probable. Este espacio se construye a partir de tres principios básicos que concretan en la práctica los principios sobre los que se sustenta cualquier proceso de IAP:

1. La co-generación del conocimiento. A partir de un proceso en el que los agentes participantes aportan y construyen conjuntamente el problema que se ha definido conjuntamente.

2. La consideración del proceso como emergente, en permanente construcción. Son los agentes los que definen el proceso en cada momento, configurándolo de forma lineal. Cada fase ayuda a definir la siguiente.

3. Respeto a la participación y aportaciones de todas las y los participantes.

Todo lo anterior nos ubica en lo que Schön (1998: 248) define como "conversación reflexiva con una situación”. Estamos ante una reflexión sobre la acción, hecha desde la acción, en diálogo permanente y sereno, que intenta situar la resolución técnica del problema objeto de estudio:

Dentro del contexto más amplio de una indagación reflexiva, [para que] muestre cómo la reflexión desde la acción puede ser rigurosa por propio derecho y vincule el arte de la práctica, en la incertidumbre y el carácter único, con el arte de la investigación del científico (ibídem: 73).

A partir de este modelo, se propone que las personas investigadoras del Departamento de Trabajo Social y Sociología de la Universidad de Deusto dinamicen este proceso, facilitando la interacción entre los agentes y ayudándoles a sistematizar sus aportaciones, favoreciendo un proceso de co-construcción orientado a buscar respuestas a los problemas que se definan conjuntamente.

En la investigación desarrollada en el marco del proyecto Gipuzkoa Sarean, Karlsen y Larrea (2015) concluyen, siguiendo a Greenwood y Levin (2007), que un proceso de este tipo se inicia cuando los 'dueños del problema' (los agentes o sujetos) deciden invitar a 'agentes externos' para iniciar un proceso de cambio. Estos agentes externos son las y los investigadores/facilitadores de un proceso de coaprendizaje orientado a la solución del problema definido por los actores.

Este proceso de participación define un modelo cíclico, co-generativo, en el que los distintos agentes identificados construyen de manera conjunta la definición y desarrollo del problema. Según los citados autores, este proceso se desarrollará a partir de las siguientes fases recogidas en la Figura 1:

- Una vez identificados las y los agentes, se inicia el proceso de definición del problema, para lo cual se desarrolla una negociación en la que, entre todas y todos, se define y prioriza el problema objeto de atención, que se convertirá en el eje a partir del cual se iniciará el trabajo.

- Tras definir el problema, se pone en marcha el espacio de reflexión y aprendizaje mutuos: es el ágora, los espacios para la reflexión y aprendizaje a partir de la generación de conocimiento mutuo desde acciones comunicativas y encuentros en un entorno estructurado. Son espacios de co-construcción que facilitarán la resolución del problema a través de la acción, y en donde las y los agentes internos implementarán acciones

Figura 1. Cambio participativo como aprendizaje co-generativo en la investigación-acción participativa

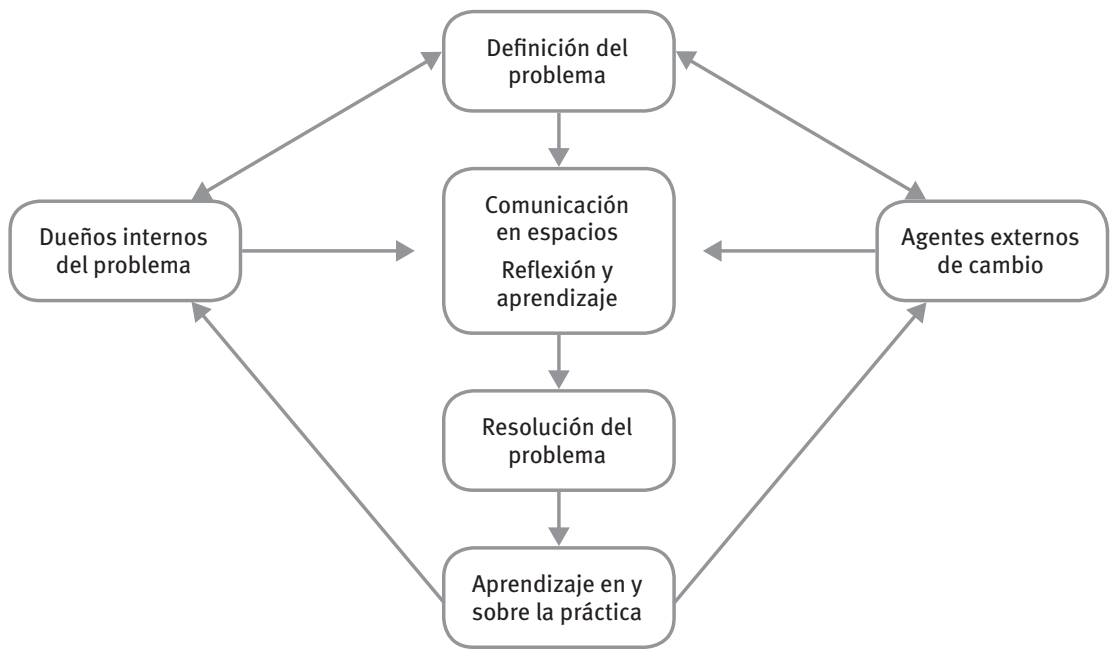

Fuente: Klev y Levin (2012), cit. en Karlsen y Larrea, 2015: 89 
tendentes a resolver los problemas tratados durante el proceso. La priorización y pilotaje de las acciones forma parte del proceso de trabajo que se diseñe a tal efecto.

- Por último, y en tanto proceso de investigaciónacción, es necesario recoger los aprendizajes obtenidos en y sobre la práctica. Así, las y los agentes internos efectuarán una evaluación de cada una de las acciones que surjan a partir del proceso, posibilitando la redefinición del problema. Las y los agentes externos, por su parte, realizarán una reflexión científicoacadémica a partir de lo experimentado en el proceso.

\subsection{Implementación del proceso}

El proceso de trabajo que estamos relatando en este artículo se inició en junio de 2016, en el marco del convenio de colaboración Etorkizuna Eraikiz (Construyendo Futuro) firmado por la DFG y la UD para realizar proyectos de investigación-acción, cuando se nos encomienda la facilitación de la gobernanza del Plan de Inclusión Social, el Plan Elkar-EKIN.

\subsubsection{Constitución del talde txikia}

Nuestro primer paso fue identificar el grupo motor del proceso, denominado talde txikia (grupo pequeño) y compuesto por personas del Departamento de Políticas Sociales de la Diputación de Gipuzkoa y por investigadoras e investigadores del Departamento de Trabajo Social y Sociología de la Universidad de Deusto, cuya función principal era liderar y dinamizar el proceso colaborativo que ayudara a establecer una nueva gobernanza entre los diversos agentes involucrados en el ámbito de la inclusión social.

Al inicio del proceso, en septiembre, el talde txikia estaba compuesto por seis personas: dos directoras/es generales del Departamento de Políticas Sociales y cuatro investigadoras/es de la universidad. En octubre, se incorporó por parte de la Diputación, una persona del Servicio de Protección a la Mujer Víctima de Violencia Machista y de Inclusión Social, quien sin embargo, por razones de índole laboral, dejó de pertenecer al grupo a final de noviembre. Por otra parte, a mediados de noviembre, hacia el final del proceso de trabajo iniciado se incorporó al grupo el jefe del citado servicio.

La identificación del talde txikia supuso una aportación relevante al esquema conceptual planteado por Karlsen y Larrea (2015), que, sin embargo, se recoge en la propuesta del Colectivo loé (2003). Su aportación es indispensable para el diseño y liderazgo del proceso que se quiere implementar. Sin embargo, cierta inestabilidad en la definición de las y los participantes del talde txikia ha supuesto una continua renegociación del encargo realizado por la Diputación (agente interno) a la universidad (agente externo), así como de las expectativas sobre las funciones de facilitación de las investigadoras y los objetivos y diseño del proceso. Esta inestabilidad, inherente a cualquier inicio de un proceso que quiere ser emergente, ha supuesto una continua revisión y renegociación de los acuerdos adoptados, que, en cualquier caso, han ayudado también a avanzar en la construcción colectiva del proceso y en la generación de una identidad como grupo.

\subsubsection{Definición del problema}

Tal y como hemos adelantado, el talde txikia asumió las funciones de diseño y liderazgo del proceso colaborativo, de modo que, en un primer momento, definió el problema objeto de trabajo y determinó quiénes eran los agentes invitados a aquél. El proceso de trabajo de identificación del problema fue arduo, debido, fundamentalmente a la indefinición del encargo y a la necesidad de conjugar los tiempos de los procesos participativos y colaborativos (lentos y a largo plazo) con los tiempos políticos (rápidos e inmediatos).

Al inicio del proceso, el talde txikia trabajó con dos perspectivas y una metáfora: había que decidir si empezar por crear 'la vasija' ('lo lento') o por crear 'el contenido', 'la excusa' para la relación ('lo rápido’):

- La metáfora de la vasija hacía alusión al espacio que aglutinara a las y los diversos agentes y posibilitara la creación de un espacio de confianza, en el que la colaboración avanzara en un plano más estratégico y menos puntual. Se trataba de trabajar para construir un clima de confianza mutua que superara prejuicios, posiciones estáticas y culturas organizacionales distintas, y que además fuera capaz de fomentar el conocimiento mutuo.

- La metáfora del contenido, o la excusa, aludía a la opción por iniciar el diálogo a partir de un asunto previamente identificado como de interés común, que respondía a las necesidades más inmediatas del Departamento foral, entendiendo que el contenido del diálogo permitiría también ir construyendo el espacio de éste (la vasija).

Por fin, el grupo motor optó por esta segunda vía, de modo que el problema identificado se formuló así:

Analizar el Servicio de Valoración de la Exclusión y la herramienta que lo debería soportar (el diagnóstico social), de forma que se pueda diseñar la ayuda económica vinculada al Plan de Atención Personalizada (PAP).

Esta decisión estuvo motivada por la necesidad de responder al anuncio político de puesta en marcha, en octubre, del Servicio Foral de Valoración de la Exclusión (competencialmente imprescindible), el cual afectaba desde el inicio a la orientación metodológica de construcción común de la definición de problema por todas y todos los agentes 
participantes del proceso. De hecho, como veremos cuando se aborde la fase del inicio del ágora, en la primera sesión de trabajo, la respuesta del resto de agentes sería reclamar la atención de otras cuestiones para ellos más urgentes que la de poner en marcha el Servicio Foral de Valoración.

\subsubsection{Identificación de los agentes internos}

Una vez concretado y definido este problema, el talde txikia identificó los agentes internos que deberían abordarlo. Se decidió convocar a las y los técnicos referentes de todos los servicios sociales municipales de Gipuzkoa, así como a las y los técnicos forales del Servicio de Protección a la Mujer Víctima de Violencia Machista y de Inclusión Social, entendiendo como prioritario iniciar las conversaciones con las y los encargados de garantizar la responsabilidad pública de la atención, dejando para un segundo momento la conversación con los agentes del tercer sector que atienden y gestionan gran parte de los recursos y programas en el ámbito de la inclusión.

En este primer proceso, participaron trabajadoras y trabajadores sociales de 22 ayuntamientos ${ }^{2}$, así como personal técnico del Servicio de Protección a la Mujer Víctima de la Violencia Machista y de Inclusión Social. Se desarrollaron tres encuentros durante los meses de octubre y noviembre de 2016.

\subsubsection{Inicio del ágora}

El objetivo del primer encuentro fue la presentación, por parte de las y los responsables del Departamento Foral de Inclusión, de la propuesta de funcionamiento del Servicio Foral de Valoración y Diagnóstico de la Exclusión, así como de la herramienta de valoración diagnóstica que estaba en fase de construcción. Igualmente se presentó la propuesta de un procedimiento experimental/piloto para desarrollarlo, en el que la propia dirección del Departamento solicitó la colaboración de las y los técnicos municipales interesados en abordar las cuestiones asociadas a este proyecto.

Como se ha adelantado anteriormente, tras las valoraciones recogidas en la primera sesión (tanto presenciales como escritas) el equipo de la Universidad de Deusto, en su función de agente externo, viendo las notables diferencias en la priorización de los asuntos/problemas a resolver en una gobernanza colaborativa del Plan de Inclusión Social, recondujo el asunto concretando más el problema objeto de trabajo, aquel en el que ambos agentes (de atención primaria -ámbito municipal-y de atención secundaria -ámbito foral-) se sienten interpelados. En este sentido, la definición del problema se concretó de la siguiente manera:

${ }^{2}$ Andoain, Astigarraga, Azkoitia, Beasain, Berastegi, Berrobi, Elduain, Bergara, San Sebastián, Eibar, Elgoibar, Hernani, Hondarribia, Irun, Irura, Soraluze, Tolosa, Usurbil, Villabona, Zarautz, Zestoa y Zumaia.
La posibilidad de construir entre todos un flujograma de cómo podría ser el itinerario de un caso gestionado de una forma colaborativa entre primaria y secundaria, desde su detección, valoración, orientación de la intervención/ itinerario, y seguimiento del mismo hasta su posible salida o no del sistema de atención.

Situamos como principios del proceso el que el trabajo desarrollado iba a tener utilidad práctica, iba a haber una escucha respetuosa y abierta al mutuo conocimiento y enriquecimiento de perspectivas, no se iban a alentar falsas expectativas de resultados, iba a darse una co-construcción de los límites del proceso y la guía del trabajo iba a ser la satisfacción de las necesidades de las personas en exclusión desde la perspectiva del ejercicio de su plena ciudadanía, sin políticas de merecimiento y validando la necesidad de revisar el binomio exigencia/ intensidad de atención. En definitiva, se trataba de que las y los profesionales de atención primaria y secundaria identificaran de forma colaborativa los nudos o dificultades existentes en el proceso de atención a las personas en situación de exclusión social, para generar buenas prácticas en el ámbito de la exclusión.

A tal fin, el equipo de facilitadores presenta un marco conceptual que permite centrar las conversaciones: se trata de un flujograma que presentamos en la Figura 2, que recoge el proceso de acceso al sistema vasco de servicios sociales, a partir de la Ley $12 / 2008$ y el Decreto 185/2015 de Cartera de Prestaciones y Servicios. Este flujograma ayudó a centrar las conversaciones, introduciendo cierto orden en las opiniones y en las deliberaciones compartidas entre las y los participantes.

En las dos sesiones celebradas, se trabajaron los siguientes aspectos:

- Sobre el acceso al sistema y las dificultades existentes en cuestiones como el empadronamiento, el trabajo con las personas usuarias del ámbito de la inclusión, el acceso desde las entidades sociales y la atención a las situaciones de urgencia.

- La valoración y el diagnóstico. Cómo se implementa en la atención primaria, cómo darle continuidad en secundaria y cómo proceder con la intervención tras la valoración foral.

- La elaboración del plan de atención personalizada (PAP) y el plan individual de atención (PIA).

- El seguimiento del caso.

- La sostenibilidad y garantía de un referente de caso a lo largo de todo el proceso colaborativamente entre niveles.

- Otros asuntos, como la coordinación; la necesidad de otros recursos, programas y servicios; la atención a las situaciones de cronicidad; y otras cuestiones necesitadas de sintonía en la definición y consenso en el abordaje. 


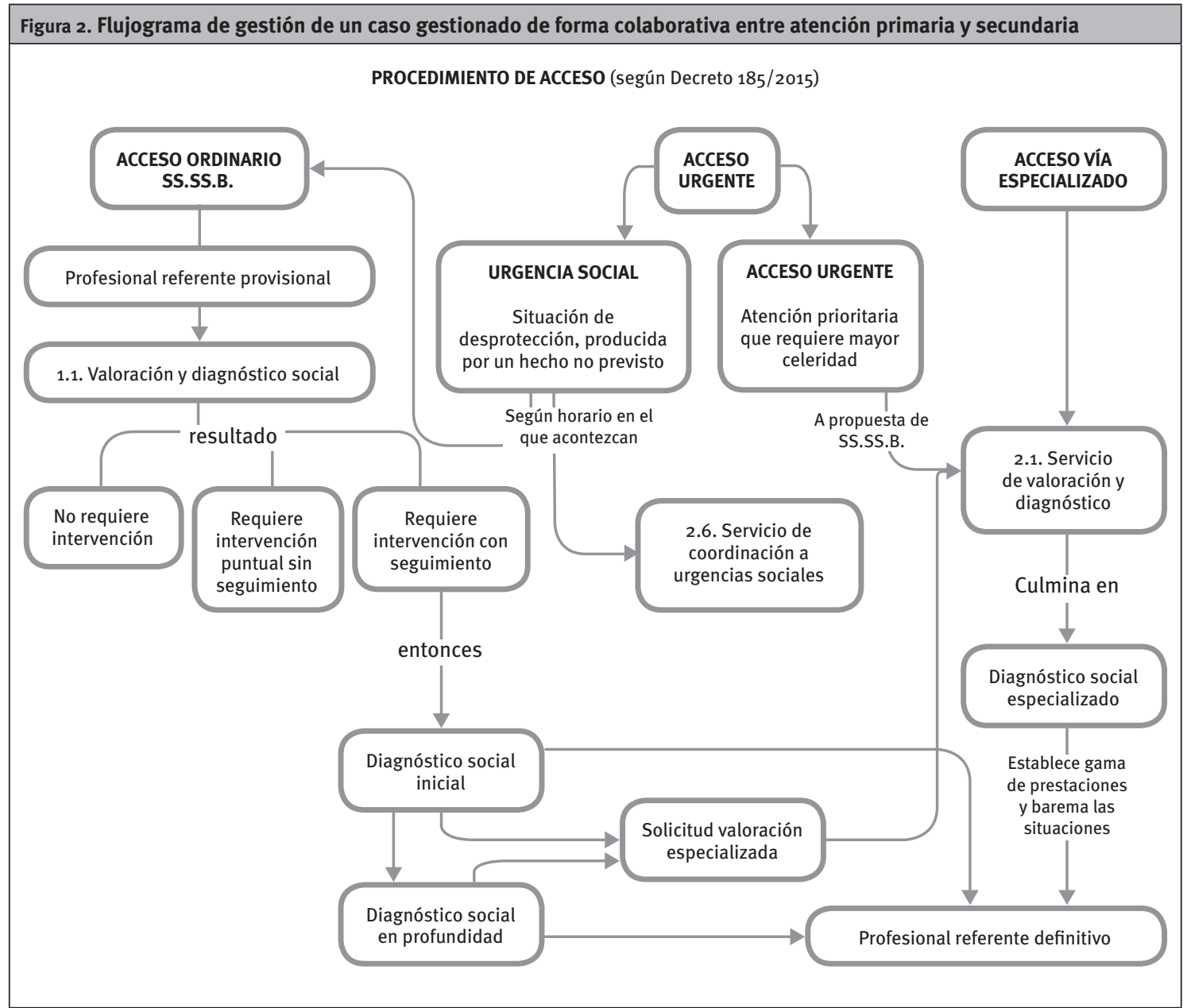

SS.SS.B.: servicios sociales de base.

Fuente: elaboración propia.

\subsubsection{Primeros pasos para la resolución del problema a través de la acción}

Lo breve de la experiencia que estamos relatando sólo nos permite señalar sobre la fase de resolución del problema el hecho de que algunas de las cuestiones planteadas en la co-construcción del flujograma han sido incorporadas al diseño y puesta en marcha del Servicio Foral de Valoración de la Exclusión, que ha iniciado su andadura en enero de 2017.

Por otra parte y como sistematización del proceso desarrollado, se ha elaborado un documento de trabajo que, como el propio proceso, tiene carácter emergente y, por tanto, inconcluso, a la espera de nuevos desarrollos y conocimientos. El documento sintetiza los pasos dados en 2016, así como las claves de la propuesta de la Diputación para implementar el Servicio de Valoración de la Exclusión. Además, sistematiza la reflexión compartida sobre el itinerario de las personas que están en riesgo o situación de exclusión, gestionado de una forma colaborativa entre primaria y secundaria. El citado documento recoge aquellas cuestiones que se entienden como problema 0 'nudos' del sistema en relación al acceso al sistema, la valoración y el diagnóstico, la elaboración del plan de atención personalizada, la realización del seguimiento, la identificación de la figura referente de caso, así como otras cuestiones de interés que, aunque no fueron abordadas, sí fueron señaladas para debates posteriores: la coordinación entre primaria y secundaria; los recursos, programas y servicios necesarios para la atención a las personas en riesgo o situación de exclusión; así como la necesidad de reflexionar sobre las situaciones de cronicidad.

\subsubsection{Inicio de la recogida de los aprendizajes obtenidos en estos primeros meses de proceso}

La evaluación de lo trabajado hasta ahora en el ágora ha permitido constatar la satisfacción de las y los participantes por disponer de un espacio de encuentro donde conversar sobre cómo responder de forma colaborativa a las necesidades de las personas en situación de exclusión, trascendiendo los debates competenciales y autorreferenciales sobre las visiones desde cada ámbito de actuación.

En lo que respecta al uso de la IAP para articular espacios de co-construcción y co-generación de 
conocimientos, nuestros aprendizajes como equipo investigador nos llevan a afirmar que:

- Es necesario establecer con mayor claridad un mapa de intereses y expectativas de los agentes internos que permita definir de manera realista nuestro papel como agentes externos facilitadores de la construcción de un nuevo modelo de gobernanza. Si, como dicen Karlsen y Larrea (2015), un proceso de investigaciónacción se inicia invitando a determinados agentes externos (en este caso, investigadores) para que participen en un proceso de cambio, es necesario que esa invitación se clarifique, acuerde y consensue. Ciertamente, y dado que estamos ante un proceso emergente, es importante gestionar la cierta inestabilidad que ello requiere.

- Consideramos importante prever con cierto realismo los tiempos necesarios para desarrollar el proceso, siguiendo las pautas metodológicas recogidas en las investigaciones contrastadas con la práctica que exponen Karlsen y Larrea (ibídem). Es fundamental dedicar tiempo y espacio a la identificación compartida del problema que se quiere resolver entre todos los agentes internos implicados. Este tiempo no es un tiempo perdido: sirve para construir el ágora, 'la vasija’ de nuestra metáfora. Sirve para establecer las pautas comunicativas que facilitarán el trabajo colaborativo, clave en un nuevo modelo de gobernanza. Obviar este paso, o no darle la importancia que tiene, supone arriesgarse a iniciar procesos que no responden a las expectativas fijadas, dificultan el cambio de posiciones y pueden profundizar las diferencias, en lugar de acercar las avenencias.

- Entendemos fundamental la presencia del talde txikia, de ese grupo motor, cuya función no es otra que la de escuchar para plantear, interpelar para avanzar, liderar para facilitar. En ningún caso debe hacerse dueño del proceso, porque no lo es. Es el medio, el instrumento para poner en palabras y en propuestas de acción los conocimientos compartidos.

- Defendemos metodologías de intervención que favorezcan las "conversaciones reflexivas con una situación”, tal y como nos propone Shön (1998). Y la IAP es clave para ello, porque permite escuchar para plantear conversaciones, en compañía de otros y otras dispuestos a dialogar para transformar.

Por todo ello y en este contexto, todos los agentes implicados en este espacio de comunicación abogamos por mantenerlo. Así, hemos identificado una nueva formulación que permitirá seguir construyendo el espacio colaborativo entre primaria y secundaria para atender mejor a las personas en situación de exclusión durante 2017, de modo que el objetivo de esta segunda fase se ha formulado de la siguiente manera:

Analizar cómo estamos dando respuesta a las necesidades de las personas en riesgo o situación de exclusión, concretamente:

- Buenas prácticas: qué estamos haciendo bien y qué hay que cuidar.

- A qué situaciones/casos no estamos dando una respuesta adecuada: motivo y alternativas.

- Impresiones sobre la implementación del nuevo Servicio de Valoración de la Exclusión.

Una vez analicemos estas cuestiones, y siguiendo con la perspectiva de la cobertura de las necesidades de las personas de manera colaborativa, identificaremos buenas prácticas en relación con la referencialidad de caso y con la implementación de los planes de atención personalizada. Por otro lado, arrancaremos con nuevos espacios de ágora, en los que incorporaremos al proceso la voz del tercer sector, en tanto agente clave en la atención a las personas que sufren situaciones de riesgo 0 exclusión en Gipuzkoa. 
ARARTEKO (2016): La situación de los servicios sociales municipales en la Comunidad Autónoma de Euskadi. Situación actual y propuesta de mejora, serie Informes Extraordinarios, VitoriaGasteiz, Ararteko.

- (2010): La situación de los servicios sociales de base en la $C A P V$, serie Informes Extraordinarios, VitoriaGasteiz, Ararteko.

ARIÑO, M.; y FERRAN, A. (2008): "Investigación - acción participación”, en Guinot, C. (coord), Métodos, técnicas y documentos utilizados en trabajo social, Bilbao, Universidad de Deusto, págs. 71-77.

BLANCO, I.; y GOMÀ, R. (2006): "Del gobierno a la gobernanza: oportunidades y retos de un nuevo paradigma”, Politika. Revista de Ciencias Sociales, $\mathrm{n}-2$, págs. 11-27.

COLECTIVO IOÉ (2003): "Investigación acción participativa: propuesta para un ejercicio activo de la ciudadanía" ['https://www.colectivoioe.org/ uploads/89050a31b85b9e19068agbeb6db3d ec136885013.pdf>].

DIPUTACIÓN FORAL DE GIPUZKOA (2015): Plan Estratégico de Gestión 2015-2019 [<http://www.gipuzkoa. eus/documents/414916/432928/Plan+Estr at $\% C_{3} \% A 9 g i c o+d e+G e s t i \% C_{3} \% B 3 n+2015-$ 2019+de+la+Diputaci\%C3\%B3n.pdf/dd86303fcoco-46d8-9526-dazedg0540cd>].

DIPUTACIÓN FORAL DE GIPUZKOA, DEPARTAMENTO DE POLÍTICAS SOCIALES (2016): Elkar-EKIN. Plan de Inclusión Social 2016-2020, Diputación Foral de Gipuzkoa [<http://www.behagi.eus/ files/informes/plan_elkar_ekin_inclusion_ gipuzkoa_2016-2020.pdf〉].

EIZAGIRRE, M.; y ZABALA, N. (2000): “Investigación-acción participativa (IAP)”, en PÉREZ DE ARMIÑO,
K. (dir.), Diccionario de acción humanitaria y cooperación al deasarrollo, Icaria; Hegoa [rhttp://www.dicc.hegoa.ehu.es/listar/ mostrar/132>].

ETXANIZ, M. (2009): “Aproximación a un modelo consensuado de servicios sociales para Gipuzkoa”, en ETXANIZ, M. et al., Ponencias del proceso de reflexión / Hausnarketa prozesuaren txostenak, Donostia-San Sebastián, Diputación Foral de Gipuzkoa, págs. 27-40.

FANTOVA, F. (2014): Diseño de políticas sociales. Fundamentos, estructura y propuestas, Madrid, CCS.

GARCÍA ROCA, J. (2006): "Memorias silenciadas en la construcción de los servicios sociales", Cuadernos de Trabajo Social, vol. 19, págs. 197-212.

JARAíZ, G. (2009): "El tercer sector de acción social en la intervención comunitaria”, Revista Española del Tercer Sector, nํㅜ 12, págs. 101-128.

KARLSEN, J.; y LARREA, M. (2015): Desarrollo territorial e investigación-acción: innovación a través del diálogo, Donostia-San Sebastián, OrkestraInstituto Vasco de Competitividad; Fundación Deusto.

PAÍS VASCO (2015): “Decreto 185/2015, de 6 de octubre, de Cartera de Prestaciones y Servicios del Sistema Vasco de Servicios Sociales", Boletín Oficial del País Vasco, no 206, 29-10-15, págs. 1-87 [<https://www.euskadi.eus/y22-bopv/es/ bopv2/datos/2015/10/1504561a.shtml>].

- (2008): "Ley 12/2008, de 5 de diciembre, de Servicios Sociales", Boletín Oficial del País Vasco, n246, 24-12-08, págs. 31.840-31.924 [<https:// www.euskadi.eus/r47-bopvapps/es/bopv2/ datos/2008/12/0807143a.shtml>]. 
- (2001): "Decreto 155/2001, de 30 de julio. 2001, de Determinación de Funciones en Materia de Servicios Sociales", Boletín Oficial del País Vasco, no 165 , 27-08-01, págs. 16.076-16.093 [khttps://www.euskadi.eus/y22-bopv/es/ bopv2/datos/2001/08/0104794a.shtmls].

SCHÖN, D. (1998): El profesional reflexivo. Cómo piensan los profesionales cuando actúan, Barcelona, Paidós.
SEN, A. (2010): Nuevo examen de la desigualdad, Madrid, Alianza.

SIIS CENTRO DE DOCUMENTACIÓN Y ESTUDIOS (2015): "Reordenación de la red de recursos y programas para la inclusión social en Gipuzkoa”, Zerbitzuan, ํํ⒌ 59, págs. 5-38 [〈https://doi.org/10.5569/1134-7147-59.01〉]. 